\title{
On the disc-convexity of complex Banach manifolds
}

\author{
by Do Duc Thai and NGuyen Le Huong (Hanoi)
}

\begin{abstract}
The Banach hyperbolicity and disc-convexity of complex Banach manifolds and their relations are investigated.

Introduction. The disc-convexity of complex Banach manifolds is one of the forms of complex convexity. It has been the object of interest for some time. Especially it was a useful tool to study the extension of holomorphic maps (see [6], [11], [13], ...).

Our aim in this article is to investigate the disc-convexity of complex Banach manifolds and the relations between the Banach hyperbolicity and disc-convexity of complex Banach manifolds.

We now describe more precisely the content of the paper.

In Section 1 we prove the existence of hyperbolic neighbourhoods of compact subsets in Banach manifolds which contain no complex lines (Proposition 1.2). As an easy corollary we prove the openness of Banach hyperbolicity for proper holomorphic maps between Banach analytic spaces (Proposition 1.6). These results are generalizations of the finite-dimensional case considered by Brody [1], Urata [16] and Zaüdenberg [18].

The above-mentioned hyperbolic neighbourhoods play a central role in our approach to disc-convexity of complex Banach manifolds.

In Section 2 we study in detail the disc-convexity of complex Banach manifolds. More precisely: we give an example of a hyperbolic and discconvex domain $S$ in $\mathbb{C}^{2}$ which is not taut (Proposition 2.2). We prove that every pseudoconvex and Brody hyperbolic (Banach) manifold is discconvex and has the Hartogs extension property (Theorem 2.3 and Proposition 2.5). Let $f: X \rightarrow Y$ be a proper holomorphic map into a Banach analytic space $Y$. If all the $f$-fibres are hyperbolic and $Y$ is discconvex, then so is $X$ (Theorem 2.6); an example is given which shows the necessity of hyperbolicity of all the $f$-fibres (Remarks 2.7.1, 2.7.2).
\end{abstract}

1991 Mathematics Subject Classification: Primary 32E05.

Key words and phrases: Banach manifold, hyperbolic, disc-convex. 
For a complex space $X$ of finite dimension define $S^{0} X=X, S^{1} X=$ $\operatorname{sing} X, \ldots, S^{i} X=\operatorname{sing} S^{i-1} X$. Then $X$ is disc-convex iff $\widetilde{S^{i} X}$ is for all $i \geq 0$ (Theorem 2.8).

Finally, the authors would like to thank the referee for many valuable comments.

1. Existence of hyperbolic neighbourhoods of compact subsets which contain no complex lines. We first give the following.

1.1. Definition. Let $X$ be a Banach $C^{k}$-manifold. We say that $X$ has $C^{k}$-partitions of unity if for every open cover $\left\{U_{i}\right\}_{i \in I}$ of $X$ there exists a family of functions $\left\{\alpha_{i}\right\}_{i \in I} \subset C^{k}(X)$ satisfying: finite.

(i) $\operatorname{supp} \alpha_{i} \subset U_{i}$ for every $i \in I$ and the family $\left\{\operatorname{supp} \alpha_{i}\right\}_{i \in I}$ is locally

(ii) $\sum_{i \in I} \alpha_{i}(x)=1$ for every $x \in X$.

The family $\left\{\alpha_{i}\right\}_{i \in I}$ is called a $C^{k}$-partition of unity subordinate to the cover $\left\{U_{i}\right\}_{i \in I}$. For details concerning smooth partitions of unity on Banach analytic manifolds we refer the readers to [15], [19]. Let $X$ be a Banach analytic space in the sense of Mazet [8]. We denote by $d_{X}$ the Kobayashi pseudodistance on $X$. In contrast to the finite-dimensional case, there exists a Banach manifold $X$ on which the Kobayashi pseudodistance is a distance but it does not define the topology of $X$. We say that $X$ is hyperbolic if $d_{X}$ is a distance defining the topology of $X$.

We now give the main result of this section.

1.2. Proposition. Let $Z$ be a compact subset in a Banach manifold $X$ having $C^{1}$-partitions of unity such that $Z$ contains no complex lines, i.e. every holomorphic map $\varphi$ from $\mathbb{C}$ into $X$ with $\varphi(\mathbb{C}) \subset Z$ is constant. Then there exists a hyperbolic neighbourhood of $Z$ in $X$.

Let $\left\{\left(U_{i}, \varphi_{i}\right)\right\}_{i \in I}$ be an atlas of $X$ such that $\varphi_{i}$ is an isomorphism from $U_{i}$ onto an open ball in Banach space for every $i \in I$. By hypothesis, there exists a $C^{1}$-partition of unity $\left\{h_{i}\right\}_{i \in I}$ subordinate to the cover $\left\{U_{i}\right\}_{i \in I}$.

Let $\pi: T X \rightarrow X$ be the tangent bundle of $X$. For each $u \in T X$, put

$$
\|u\|=\sum h_{i}(\pi u)\left\|D \varphi_{i}(\pi u)(u)\right\| .
$$

Denote by $\varrho_{X}$ the integral distance on $X$ associated with $\|\cdot\|$.

1.3. Lemma. $\varrho_{U}$ defines the topology in $U$, where $U$ is the unit ball in a Banach space $B$.

Proof. By [12] we have

$$
\|x-y\|=\sup \left\{\left|x^{*}(x)-x^{*}(y)\right|: x^{*} \in B^{*},\left\|x^{*}\right\| \leq 1\right\}
$$




$$
\begin{aligned}
& \leq \sup \left\{|f(x-y)|: f \in H^{\infty}(U), f(0)=0,\|f\| \leq 1\right\} \\
& =\frac{e^{2 c_{U}(x, y)}-1}{e^{2 c_{U}(x, y)}+1}
\end{aligned}
$$

where $B^{*}$ denotes the dual space of $B$ and $c_{U}$ denotes the Carathéodory distance on $U$. On the other hand, for the differential Carathéodory metric $\gamma_{U}$ of $U$ we have by [19],

$$
\begin{aligned}
c_{U}(x, y) & \leq \inf \left\{\int_{0}^{1} \gamma_{U}(\dot{\sigma}(t)) d t: \sigma \in \Omega_{x, y}(U)\right\} \\
& \leq \inf \left\{\int_{0}^{1}\|\dot{\sigma}(t)\| d t: \sigma \in \Omega_{x, y}(U)\right\}=\varrho_{U}(x, y)
\end{aligned}
$$

for all $x, y \in U$, where $\Omega_{x, y}$ is the set of $C^{1}$-paths joining $x$ and $y$ in $U$. Thus $\varrho_{U}$ defines the topology of $U$.

1.4. Lemma. Assume that $X$ is a Banach manifold having $C^{1}$-partitions of unity. Then $\varrho_{X}$ defines the topology of $X$.

Proof. Let $\left\{x_{n}\right\} \subset X$ and $\varrho_{X}\left(x_{n}, x\right) \rightarrow 0$. Take $j_{0}$ such that $x \in U_{j_{0}}$ and $h_{j_{0}}(x) \neq 0$. For each $n \geq 1$ take $\sigma_{n} \in \Omega_{x_{n}, x}(X)$ such that

$$
\begin{aligned}
\varrho_{X}\left(x_{n}, x\right) & \geq \int_{0}^{1}\left\|\dot{\sigma}_{n}(t)\right\| d t-1 / n \\
& =\int_{0}^{1} \sum_{j} h_{j}\left(\sigma_{n}(t)\right)\left\|D \varphi_{j}\left(\sigma_{n}(t)\right)\left(\dot{\sigma}_{n}(t)\right)\right\| d t-1 / n \\
& \geq \int_{0}^{1} h_{j_{0}}\left(\sigma_{n}(t)\right)\left\|D \varphi_{j_{0}}\left(\sigma_{n}(t)\right)\left(\dot{\sigma}_{n}(t)\right)\right\| d t-1 / n .
\end{aligned}
$$

Assume that $x_{n} \nrightarrow x$. Then there exists an open neighbourhood $V$ of $x$ in $U_{j_{0}}$ such that $x_{n} \notin V$ for all $n \geq 1$ and $\inf \left\{h_{j_{0}}(y): y \in V\right\}=\varepsilon>0$. For every $n \geq 1$, put $\varepsilon_{n}=\sup \left\{r>0: \sigma_{n}([0, r]) \subset V\right\}>0$. We have

$$
\begin{aligned}
\varrho_{X}\left(x_{n}, x\right) & \geq \int_{0}^{1} h_{j_{0}}\left(\sigma_{n}(t)\right)\left\|D \varphi_{j_{0}}\left(\sigma_{n}(t)\right)\left(\dot{\sigma}_{n}(t)\right)\right\| d t-1 / n \\
& \geq \varepsilon \int_{0}^{\varepsilon_{n}} h_{j_{0}}\left(\sigma_{n}(t)\right)\left\|D \varphi_{j_{0}}\left(\sigma_{n}(t)\right)\left(\dot{\sigma}_{n}(t)\right)\right\| d t-1 / n \\
& \geq \varepsilon \int_{0}^{\varepsilon_{n}}\left\|D \varphi_{j_{0}}\left(\sigma_{n}(t)\right)\left(\dot{\sigma}_{n}(t)\right)\right\| d t-1 / n
\end{aligned}
$$




$$
\begin{aligned}
& =\varepsilon \int_{0}^{1}\left\|D \varphi_{j_{0}}\left(\beta_{n}(s)\right)\left(\dot{\beta}_{n}(s)\right)\right\| d s-1 / n \\
& \geq \varrho_{\varphi_{j_{0}}\left(U_{j_{0}}\right)}\left(\varphi_{j_{0}}\left(\beta_{n}(1)\right), \varphi_{j_{0}}(x)\right)-1 / n
\end{aligned}
$$

where $s=t / \varepsilon_{n}$ and $\beta_{n}(s)=\sigma_{n}\left(\varepsilon_{n} s\right)$ for $s \in[0,1]$. It follows that $\left.\varrho_{\varphi_{j_{0}}\left(U_{j_{0}}\right)}\left(\varphi_{j_{0}}\left(\beta_{n}(1)\right), \varphi_{j_{0}}(x)\right)\right) \rightarrow 0$. By Lemma $1.3, \varphi_{j_{0}}\left(\beta_{n}(1)\right) \rightarrow \varphi_{j_{0}}(x)$. Hence $\beta_{n}(1) \rightarrow x$. This is a contradiction because $\beta_{n}(1)=\sigma_{n}\left(\varepsilon_{n}\right) \in \partial V$ for every $n \geq 1$.

1.5. Lemma. Let $X$ be a Banach manifold having $C^{1}$-partitions of unity such that

$$
\sup \left\{\left\|f^{\prime}(0)\right\|: f \in \operatorname{Hol}(\Delta, X)\right\}<\infty,
$$

where $\operatorname{Hol}(\Delta, X)$ denotes the space of holomorphic maps from the open unit disc $\Delta$ in $\mathbb{C}$ into $X$. Then $X$ is hyperbolic.

Proof. Let $d_{X}\left(x_{n}, x\right) \rightarrow 0$. For each $n \geq 1$ there exists a holomorphic chain $\left(f_{1}^{n}, \ldots, f_{k_{n}}^{n}, a_{1}^{n}, \ldots, a_{k_{n}}^{n}\right)$ joining $x_{n}$ and $x$ such that

$$
\sum_{j=1}^{k_{n}} d_{\Delta}\left(0, a_{j}^{n}\right) \rightarrow 0
$$

By the hypothesis we have

$$
a=\sup \left\{\left\|f^{\prime}(z)\right\|: f \in \operatorname{Hol}(\Delta, X),|z|<r\right\}<\infty,
$$

where $0<r<1$ is chosen such that $\left|a_{j}^{n}\right|<r$ for $j=1, \ldots, k_{n}$. Then

$$
\varrho_{X}\left(p_{i}^{n}, p_{i-1}^{n}\right) \leq \int_{0}^{1}\left\|\left(f_{j}^{n} \sigma_{i}^{n}\right)^{\prime}(t)\right\| d t \leq a \int_{0}^{1}\left\|\dot{\sigma}_{i}^{n}(t)\right\| d t=a d_{\Delta}\left(0, a_{i}^{n}\right),
$$

where $p_{i}^{n}=f_{i}^{n}\left(a_{i}^{n}\right)$ and $\sigma_{i}^{n}(z)=a_{i}^{n} z$. Thus $\varrho_{X}\left(x_{n}, x\right) \rightarrow 0$. By Lemma 1.4 we have $x_{n} \rightarrow x$.

Proof of Proposition 1.2. By Lemma 1.5 it suffices to show that there exists a neighbourhood $W$ of $Z$ in $X$ such that

$$
\sup \left\{\left\|f^{\prime}(0)\right\|: f \in \operatorname{Hol}(\Delta, W)\right\}<\infty .
$$

If not, for each $n$ we can find $f_{n} \in \operatorname{Hol}\left(\Delta, W_{n}\right)$ such that $\left\|f_{n}^{\prime}(0)\right\|=r_{n} \uparrow$ $\infty$, where $\left\{W_{n}\right\}$ is decreasing neighbourhood basis of $Z$ in $X$. By the parametrization lemma of Brody [1] there exists for each $n$ a holomorphic map $\varphi_{n}$ from $\left(r_{n} / 2\right) \Delta$ into $W_{n}$ such that $\left\|\varphi_{n}^{\prime}(0)\right\|=1$ and

$$
\left\|\varphi_{n}^{\prime}(z)\right\| \leq \frac{r_{n}^{2}}{r_{n}^{2}-|z|^{2}} \quad \text { for }|z|<r_{n} / 2 .
$$

This yields $\left\|\varphi_{n}^{\prime}(z)\right\| \leq 4 / 3$ for $|z|<r_{n} / 2$ and hence $\left\{\varphi_{n}\right\}$ is equicontinuous. By the compactness of $Z$ and since $Z=\bigcap W_{n}$, it follows that $\left\{\varphi_{n}\right\}$ contains 
a subsequence $\left\{\psi_{n}\right\}$ converging to $\psi \in \operatorname{Hol}(\mathbb{C}, X)$ with $\psi(\mathbb{C}) \subset Z$. Obviously, $\psi \neq$ const. This is impossible because $Z$ contains no complex lines.

1.6. Proposition. Let $\theta: X \rightarrow Y$ be a proper holomorphic map from a Banach manifold $X$ having $C^{1}$-partitions of unity into a Banach analytic space $Y$. Assume that $\theta^{-1}\left(y_{0}\right)$ is hyperbolic for some $y_{0} \in Y$. Then there exists an open neighbourhood $U$ of $y_{0}$ in $Y$ such that $\theta^{-1}(U)$ is Banach hyperbolic.

Proof. By Proposition 1.2, there exists a hyperbolic neighbourhood $W$ of $\theta^{-1}\left(y_{0}\right)$ in $X$.

Suppose that there is no neighbourhood $U$ of $y_{0}$ in $Y$ such that $\theta^{-1}(U)$ $\subset W$. Consider a decreasing sequence $\left\{U_{n}\right\}$ of neighbourhoods of $y_{0}$ which is convergent to $y_{0}$. Then for each $n \geq 1$ there are $y_{n} \in U_{n}$ and $x_{n} \in \theta^{-1}\left(y_{n}\right)$ such that $x_{n} \notin W$. Since the set $K=\left\{y_{n}: n \geq 1\right\} \cup\left\{y_{0}\right\}$ is compact, so is

$$
\theta^{-1}(K)=\bigcup_{n \geq 1} \theta^{-1}\left(y_{n}\right) \cup \theta^{-1}\left(y_{0}\right) \text {. }
$$

Thus $\left\{x_{n}\right\}$ contains subsequence $\left\{x_{n_{k}}\right\}$ convergent to $x_{0}$. It follows that $\theta x_{n_{k}} \rightarrow \theta x_{0}$, i.e. $y_{n_{k}} \rightarrow \theta x_{0}$. Hence $\theta x_{0}=y_{0}$, i.e. $x_{0} \in \theta^{-1}\left(y_{0}\right) \subset W$. Thus $x_{n_{k}} \in W$ for all $k \geq N$. This is impossible, because $x_{n} \notin W$ for each $n \geq 1$.

1.7. Remark. From a result of Ramis [9, Théorème II.2.1.1, p. 36], we can deduce the existence of finite proper holomorphic maps between Banach analytic spaces.

Let $S$ be an analytic subset of codimension $p$ in a Banach space $E$. Then we can decompose $E=B \oplus \mathbb{C}^{p}$ so that the restriction $\left.\pi\right|_{S}$ of the canonical projection $\pi: E \rightarrow B$ is a finite proper holomorphic map from $S$ onto $B$ (at least locally).

2. Disc-convexity of Banach analytic spaces. We first give the following.

2.1. Definitions. For every $0<r<1$ and $s>0$ we define

$$
\Delta_{s}=\{z \in \mathbb{C}:|z|<s\}, \quad \Delta_{1}=\Delta, \quad \Delta_{r 1}=\{z \in \mathbb{C}: r<|z|<1\} .
$$

We say that a Banach analytic space $X$ is disc-convex if every sequence $\left\{f_{n}\right\} \subset \operatorname{Hol}(\Delta, X)$ converges in $\operatorname{Hol}(\Delta, X)$ whenever $\left\{\left.f_{n}\right|_{\Delta_{r 1}}\right\}$ converges in $\operatorname{Hol}\left(\Delta_{r 1}, X\right)$ for some $r<1$, where $\operatorname{Hol}(X, Y)$ denotes the space of all holomorphic maps from $X$ into $Y$ with the open-compact topology. It is well known that, by the Montel theorem, a taut (finite-dimensional) complex space is disc-convex and hyperbolic. The converse is not true in general.

Let $u(z)$ be a negative subharmonic function on $\Delta$. In addition, suppose $u(z)$ is bounded from below and discontinuous at 0 and $\lim _{z \rightarrow 0} u(z)<u(0)$. 
In $\mathbb{C}^{2}$ we consider the Hartogs domain

$$
S=\left\{(z, w) \in \mathbb{C}^{2}:|z|<1,|w|<e^{-u(z)}\right\}
$$

(see Shabat [10] or Diederich and Sibony [2]).

We have the following.

2.2. Proposition. The domain $S$ is hyperbolic and disc-convex but not taut.

Pr o of. Clearly $S$ is bounded and by the Hartogs theorem, $S$ is a domain of holomorphy. Then $S$ is a hyperbolic disc-convex manifold.

Now assume that $S$ is taut. Put $-\lim _{z \rightarrow 0} u(z)=R>-u(0)$. Take $\beta \in \mathbb{R}$ and a sequence $\left\{z_{n}\right\} \subset \Delta$ converging to 0 such that

$$
\lim _{n \rightarrow \infty}\left(-u\left(z_{n}\right)\right)=R>\ln \beta>-u(0) .
$$

Without loss of generality we can suppose that

$$
-u\left(z_{n}\right)>\ln \beta>-u(0) \quad \text { for all } n \geq 1 .
$$

Let $\theta: \Delta_{\beta} \rightarrow \Delta_{\beta}$ be a biholomorphic map such that $\theta(0)=e^{-u(0)}$. Then the map $f: \Delta \rightarrow \Delta_{\beta}$, where $f(z)=\theta(\beta z)$ for all $z \in \Delta$, is biholomorphic and $f(\Delta) \subset \Delta_{e^{-u\left(z_{n}\right)}}$ for all $n \geq 1$.

Consider holomorphic maps $g_{n}: \Delta \rightarrow S, z \mapsto\left(z_{n}, f(z)\right)$, for all $n \geq 1$. We have $\lim _{n \rightarrow \infty} g_{n}(z)=(0, f(z))$ for all $z \in \Delta$ and $\lim _{n \rightarrow \infty} g_{n}(0)=$ $(0, f(0)) \notin S$. Since $S$ is taut, $(0, f(z)) \notin S$ for all $z \in \Delta$. Hence $|f(z)| \geq$ $e^{-u(0)}=f(0)$ for all $z \in \Delta$. This is impossible.

2.3. Theorem. Let $X$ be a pseudoconvex Banach manifold having $C^{1}$ partitions of unity. Suppose that $X$ contains no complex lines. Then $X$ is disc-convex.

Pr o of. Assume that a sequence $\left\{f_{n}\right\} \subset \operatorname{Hol}(\Delta, X)$ is such that $\left\{\left.f_{n}\right|_{\Delta_{r 1}}\right\}$ converges, uniformly on compact sets, to a map $f$ in $\operatorname{Hol}\left(\Delta_{r 1}, X\right)$. Let $\left\{f_{n_{k}}\right\}$ be any subsequence of $\left\{f_{n}\right\}$. Put $K=\bigcup_{k=1}^{\infty} f_{n_{k}}\left(\partial \Delta_{s}\right)$, where $r<s<1$.

By the hypothesis and by the maximum principle it follows that $(\bar{K})_{\mathrm{PSH}(X)}^{\wedge}$ is compact and

$$
\bigcup_{k=1}^{\infty} f_{n_{k}}\left(\Delta_{s}\right) \subset(\bar{K})_{\operatorname{PSH}(X)}^{\wedge}
$$

Again by Proposition 1.2, there is a hyperbolic neighbourhood $W$ of $(\bar{K})_{\mathrm{PSH}(X)}^{\wedge}$ in $X$. This implies that the family $\left\{f_{n_{k}}\right\}$ is equicontinuous. On the other hand, since $\left\{f_{n_{k}}(\lambda)\right\}$ is relatively compact for each $\lambda \in \Delta_{s}$, by the Ascoli theorem the family $\left\{f_{n_{k}}: k \geq 1\right\}$ is relatively compact in $\operatorname{Hol}\left(\Delta_{s}, X\right)$. Thus there exists a subsequence $\left\{f_{n_{k_{l}}}\right\}$ of $\left\{f_{n_{k}}\right\}_{k=1}^{\infty}$ which converges, uniformly on compact sets, to the map $F$ in $\operatorname{Hol}(\Delta, X)$. The equality $\left.F\right|_{\Delta_{r 1}}=f$ determines $F$ uniquely, hence independently of the choices of the subse- 
quences $\left\{f_{n_{k}}\right\}$. It follows that $\left\{f_{n}\right\}$ converges, uniformly on compact sets, to the map $F$ in $\operatorname{Hol}(\Delta, X)$.

We now recall the following definition.

2.4. Definition. Let $X$ be a Banach analytic space. We say that $X$ has the Hartogs extension property (briefly HEP) if every holomorphic map from a Riemann domain over a Banach space having a Schauder basis into $X$ can be extended to the envelope of holomorphy of that map.

The following assertion is deduced immediately from Theorem 2.3 and the result of B. D. Tac [13].

2.5. Proposition. Let $X$ be a pseudoconvex Banach manifold having $C^{1}$-partitions of unity. If $X$ contains no complex line then $X$ has HEP.

2.6. Theorem. Let $\pi: X \rightarrow Y$ be a proper holomorphic map from a Banach manifold $X$ having $C^{1}$-partitions of unity onto a Banach analytic space $Y$ such that the fibre $\pi^{-1}(y)$ is hyperbolic for all $y \in Y$. If $Y$ is disc-convex, then so is $X$.

Pr o of. Assume that $\left\{f_{n}\right\} \subset \operatorname{Hol}(\Delta, X)$ is a sequence such that $\left\{\left.f_{n}\right|_{\Delta_{r 1}}\right\}$ converges, uniformly on compact sets, to a map $f$ in $\operatorname{Hol}\left(\Delta_{r 1}, X\right)$. Let $\left\{f_{n_{k}}\right\}$ be any subsequence of $\left\{f_{n}\right\}$. Put $g_{k}=\pi \circ f_{n_{k}}$ for all $k \geq 1$. Since $Y$ is disc-convex, $\left\{g_{k}\right\}$ converges uniformly to a map $G$ in $\operatorname{Hol}(\Delta, X)$.

Consider the family $\mathcal{V}$ of all pairs $(V, F)$, where $V$ is an open subset of $\Delta$ containing $\Delta_{r 1}$ and $F \in \operatorname{Hol}(V, X)$ is such that there exists a subsequence $\left\{\left.f_{n_{k_{l}}}\right|_{V}\right\}$ of $\left\{\left.f_{n_{k}}\right|_{V}\right\}$ converging, uniformly on compact sets, to $F$ in $\operatorname{Hol}(V, X)$. We define an order relation in the family $\mathcal{V}$ by $\left(V_{1}, F_{1}\right) \leq\left(V_{2}, F_{2}\right)$ if $V_{1} \subset V_{2}$ and for every subsequence $\left\{\left.f_{n_{k_{1}}}\right|_{V_{1}}\right\}$ of $\left\{\left.f_{n_{k}}\right|_{V_{1}}\right\}$ converging, uniformly on compact sets, to $F_{1}$ in $\operatorname{Hol}\left(V_{1}, X\right)$, the sequence $\left\{\left.f_{n_{k_{1}}}\right|_{V_{2}}\right\}$ contains a subsequence converging, uniformly on compact sets, to $F_{2}$ in $\operatorname{Hol}\left(V_{2}, X\right)$.

Assume that $\left\{\left(V_{\alpha}, F_{\alpha}\right)\right\}_{\alpha \in \Lambda}$ is a well-ordered subset of $\mathcal{V}$. Put $V_{0}=$ $\bigcup_{\alpha \in \Lambda} V_{\alpha}$. Define a map $F_{0} \in \operatorname{Hol}\left(V_{0}, X\right)$ by $\left.F_{0}\right|_{V_{\alpha}}=F_{\alpha}$ for all $\alpha \in \Lambda$. Take a sequence $\left\{\left(V_{i}, F_{i}\right)\right\}_{i=1}^{\infty} \subset\left\{\left(V_{\alpha}, F_{\alpha}\right)\right\}_{\alpha \in \Lambda}$ such that

$$
\left(V_{1}, F_{1}\right) \leq\left(V_{2}, F_{2}\right) \leq \ldots \text { and } \bigcup_{i=1}^{\infty} V_{i}=V_{0} .
$$

By assumption, there is a subsequence $\left\{\left.f_{k}^{1}\right|_{V_{1}}\right\}$ of $\left\{\left.f_{n_{k}}\right|_{V_{1}}\right\}$ converging to $F_{1}$ in $\operatorname{Hol}\left(V_{1}, X\right)$. Consider the sequence $\left\{\left.f_{k}^{1}\right|_{V_{2}}\right\}$. As above, it contains a subsequence $\left\{\left.f_{k}^{2}\right|_{V_{2}}\right\}$ converging to $F_{2}$ in $\operatorname{Hol}\left(V_{2}, X\right)$. Continuing this process we can find sequences $\left\{f_{k}^{i}\right\}$ such that $\left\{f_{k}^{i}\right\} \subset\left\{f_{k}^{i-1}\right\}$ for all $i \geq 2$ and $\left\{\left.f_{k}^{i}\right|_{V_{i}}\right\}$ converges to $F_{i}$ in $\operatorname{Hol}\left(V_{i}, X\right)$. Then the sequence $\left\{f_{i}^{i}\right\}$ converges to $F_{0}$ in 
$\operatorname{Hol}\left(V_{0}, X\right)$. Thus $\left(V_{0}, F_{0}\right) \in \mathcal{V}$ and hence, the subset $\left\{\left(V_{\alpha}, F_{\alpha}\right)\right\}_{\alpha \in \Lambda}$ has an upper bound.

By the Zorn lemma, the family $\mathcal{V}$ has a maximal element $(V, F)$. Let $\left\{\left.F_{n_{k_{l}}}\right|_{V}\right\}$ be a subsequence of $\left\{\left.f_{n_{k}}\right|_{V}\right\}$ converging uniformly to $F$ in $\operatorname{Hol}(V, X)$. We now prove that $V$ is closed in $\Delta$.

Indeed, take $z_{0} \in V$. By Proposition 1.6, there is an open neighbourhood $U$ of $G\left(z_{0}\right)$ in $Y$ such that $\pi^{-1}(U)$ is hyperbolic. Since $\left\{g_{k}\right\}$ converges uniformly to $G$ in $\operatorname{Hol}(\Delta, Y)$, there is an open neighbourhood $W$ of $z_{0}$ in $\Delta$ such that $g_{k}(W) \subset U$ for all $k \geq N$. Hence $f_{n_{k}}(W) \subset \pi^{-1}(U)$ for all $k \geq N$. Since $\pi$ is a proper map, $\left\{f_{n_{k_{l}}}(z): l \geq 1\right\}$ is relatively compact in $\pi^{-1}(U)$ for all $z \in W$. By the equicontinuity of $\left\{f_{n_{k_{l}}}\right\}$ for $d_{\pi^{-1}(U)}$, the family $\left\{f_{n_{k_{l}}}: l \geq 1\right\}$ is relatively compact in $\operatorname{Hol}\left(W, \pi^{-1}(U)\right)$. By the maximality of the element $(V, F)$, we have $W \subset V$ and hence, $V=\Delta$.

Thus the sequence $\left\{f_{n_{k_{l}}}\right\}$ converges, uniformly on compact sets, to the map $F$ in $\operatorname{Hol}(\Delta, X)$. The equality $\left.F\right|_{\Delta_{r 1}}=f$ determines $F$ uniquely, hence independently of the choices of the subsequences $\left\{f_{n_{k}}\right\}$. It follows that $\left\{f_{n}\right\}$ converges, uniformly on compact sets, to $F$ in $\operatorname{Hol}(\Delta, X)$.

2.7. REMARK. 1. The following counterexample shows that the condition of hyperbolicity of all fibres in Theorem 2.6 cannot be replaced by the condition of disc-convexity of all fibres. Consider the canonical holomorphic map $\theta$ from the Hopf surface $X=\mathbb{C} \backslash\{0\} /(z \sim 2 z)$ onto $\mathbb{C} P^{1}$. Then $\theta^{-1}(y) \cong \mathbb{C} \backslash\{0\} /(z \sim 2 z)$. Since the universal cover of $\mathbb{C} \backslash\{0\} /(z \sim 2 z)$ is a Stein manifold, every fibre $\theta^{-1}(y) \cong \mathbb{C} \backslash\{0\} /(z \sim 2 z)$, which is an elliptic curve, satisfies the condition of disc-convexity.

We check that there exists a non-empty open subset $V$ of $\mathbb{C} P^{1}\left(V \neq \mathbb{C} P^{1}\right)$ such that $\theta^{-1}(V)$ is not disc-convex.

Otherwise consider the commutative diagram

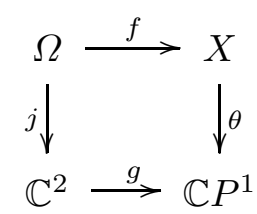

in which $\Omega=\mathbb{C}^{2} \backslash\{0\} ; f: \Omega \rightarrow X$ is the canonical map; $\widetilde{\Omega}$ is the local envelope of biholomorphy of $f$ over $\mathbb{C}^{2}$, the envelope of holomorphy of $\mathbb{C}^{2} \backslash$ $\{0\} ; g: \mathbb{C}^{2} \rightarrow \mathbb{C} P^{1}$ is the meromorphic extension of $\theta f$; and $j, \theta$ are canonical maps.

It is easy to see that $\widetilde{f}: \widetilde{\Omega} \rightarrow X$ is locally pseudoconvex, i.e. for every $x \in X$ there is a pseudoconvex neighbourhood $U$ of $x$ in $X$ such that $\widetilde{f}^{-1}(U)$ is pseudoconvex. By hypothesis, $\theta \widetilde{f}: \widetilde{\Omega} \rightarrow \mathbb{C} P^{1}$ is locally pseudoconvex. 
Since $X$ is homogeneous compact, as follows from [4], e: $\Omega \rightarrow \widetilde{\Omega}$ and hence $f: \Omega \rightarrow X$ extends holomorphically to $\mathbb{C}^{2}$. This is impossible.

2. The disc-convexity is not closed under proper holomorphic maps. Indeed, let $Z=\left\{(z,[w]) \in \mathbb{C} \times \mathbb{C} \backslash\{0\} /(z \sim 2 z): z_{i} w_{j}=z_{j} w_{i}, 1 \leq i, j \leq 2\right\}$ and $\theta: Z \rightarrow \mathbb{C}^{2}$ be the canonical projection. We have

$$
\theta^{-1}(z)= \begin{cases}\mathbb{C}^{2} \backslash\{0\} /(z \sim 2 z) & \text { if } z=0 \\ \mathbb{C} \backslash\{0\} /(z \sim 2 z) & \text { if } z \neq 0\end{cases}
$$

Clearly, $\theta^{-1}(z)$ is disc-convex for each $z \neq 0$ but $\theta^{-1}(0)$ is not disc-convex.

We now investigate the disc-convexity of the normalizations of complex spaces.

2.8. Theorem. Let $X$ be a (finite-dimensional) complex space. Then $X$ is disc-convex if and only if $\widetilde{S^{i} X}$ is disc-convex for all $i \geq 0$, where $S^{0} X=X, S^{1} X=S(X)$ is the singular locus of $X$, and $S^{i} X=S\left(S^{i-1} X\right)$ for all $i \geq 2$.

Proof. Let $X$ be a (finite-dimensional) disc-convex space. Then $S^{i} X$ is disc-convex for every $i \geq 0$. By Theorem 2.6, $\widehat{S^{i} X}$, the normalization of $S^{i} X$, is also disc-convex for every $i \geq 0$.

Now assume that $\widetilde{S^{i} X}$ is disc-convex for every $i \geq 0$. Given $g \in$ $\operatorname{Hol}(\Delta, X)$, take $i \geq 0$ such that $g(\Delta) \subset S^{i} X$ but $g(\Delta) \not \subset S^{i+1} X$. Consider the diagram

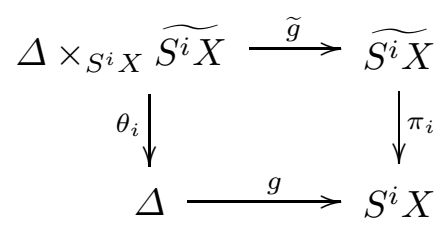

Since $\pi_{i}$ is finite and proper, so is $\theta_{i}$. By the normality of $\Delta$ and by the integrity lemma [3], it follows that $\theta_{i}: \Delta \times_{S^{i} X} S^{i} X \rightarrow \Delta$ is an analytic covering map. This yields that card $\theta_{i}^{-1}(z)=1$ for every $z \in \Delta$. Hence from the normality of $\Delta$ we deduce that $\widetilde{g} \circ \theta_{i}^{-1}: \Delta \rightarrow \widetilde{S^{i} X}$ is holomorphic.

Let $\left\{\varphi_{j}\right\} \subset \operatorname{Hol}(\Delta, X)$ be a sequence such that the sequence $\left\{\left.\varphi_{j}\right|_{\Delta_{r 1}}\right\}$ converges, uniformly on compact sets, to a map $f$ in $\operatorname{Hol}\left(\Delta_{r 1}, X\right)$ for some $0<r<1$. Let $\left\{\varphi_{j_{n}}\right\}$ be any subsequence of $\left\{\varphi_{j}\right\}$. Put $\varphi_{j_{n}}=f_{n}$ for all $n \geq 1$. Then we can find $i \geq 0$ and a subsequence $\left\{f_{n_{k}}\right\}$ of $\left\{f_{n}\right\}$ such that $f_{n_{k}}(\Delta) \subset S^{i} X$ but $f_{n_{k}}(\Delta) \not \subset S^{i+1} X$ for all $k \geq 1$. Since $S^{i} X$ is closed in $X$, $f\left(\Delta_{r 1}\right) \subset S^{i} X$. 
Consider the commutative diagram

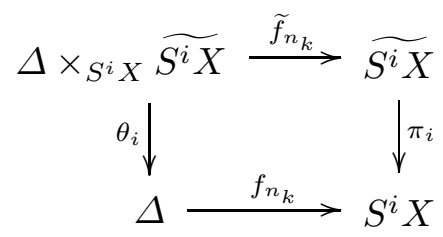

Reasoning as above, we deduce that $g_{k}=\widetilde{f}_{n_{k}} \circ \theta_{i}^{-1}: \Delta \rightarrow \widetilde{S^{i} X}$ is holomorphic for every $k \geq 1$. Take any point $z \in \Delta_{r 1}$. By the results of Brody [1], Urata [16] and Zaldenberg [18], there is a complete hyperbolic neighbourhood $U$ of $f(z)$ in $S^{i} X$ such that $\pi_{i}^{-1}(U)$ is complete hyperbolic. Since the sequence $\left\{\left.f_{n_{k}}\right|_{\Delta_{r 1}}\right\}$ converges uniformly to the map $f$ in $\operatorname{Hol}\left(\Delta_{r 1}, S^{i} X\right)$, there is an open neighbourhood $V$ of $z$ in $\Delta_{r 1}$ such that $f_{n_{k}}(V) \subset U$ for all $k \geq N$. Hence $g_{k}(V) \subset \pi_{i}^{-1}(U)$ for all $k \geq N$.

Suppose that the sequence $\left\{\left.g_{k}\right|_{V}\right\}$ contains a subsequence which is compactly divergent. Without loss of generality we may assume that $\left\{\left.g_{k}\right|_{V}\right\}$ itself is compactly divergent. Let $K$ and $L$ be two compact subsets in $V$ and $U$ respectively. Since $\pi_{i}^{-1}(L)$ is compact, there is $k_{0}$ such that $g_{k}(K) \cap \pi_{i}^{-1}(L)=\emptyset$ for all $k>k_{0}$. This implies that $f_{n_{k}}(K) \cap L=\emptyset$ for all $k>k_{0}$, and hence the sequence $\left\{\left.f_{n_{k}}\right|_{V}\right\}$ is compactly divergent. This is impossible.

Thus $\left\{\left.g_{k}\right|_{V}\right\}$ contains a subsequence which is uniformly convergent in $\operatorname{Hol}\left(V, \widetilde{S^{i} X}\right)$. Repeating the proof of Theorem 2.6 we can find a subsequence $\left\{g_{k_{l}}\right\}$ of $\left\{g_{k}\right\}$ such that $\left\{\left.g_{k_{l}}\right|_{\Delta_{r 1}}\right\}$ converges uniformly to a map $G$ in $\operatorname{Hol}\left(\Delta_{r 1}, \widetilde{S^{i} X}\right)$. Since $\widetilde{S^{i} X}$ is disc-convex, $\left\{g_{k_{l}}\right\}$ converges uniformly to a map $\widetilde{G} \in \operatorname{Hol}\left(\Delta, \widetilde{S^{i} X}\right)$ in $\operatorname{Hol}\left(\Delta, \widetilde{S^{i} X}\right)$. Hence $\left\{f_{n_{k_{l}}}\right\}$ converges uniformly to $\pi_{i} \circ \widetilde{G}=F$ in $\operatorname{Hol}(\Delta, X)$. The equality $\left.F\right|_{\Delta_{r 1}}=f$ determines $F$ uniquely, hence independently of the choices of the subsequences $\left\{\varphi_{j_{n}}\right\}$. It follows that $\left\{\varphi_{j}\right\}$ converges, uniformly on compact sets, to the map $F$ in $\operatorname{Hol}(\Delta, X)$.

Note that the analogous result for the tautness of normalizations of complex spaces was proved in [14].

\section{References}

[1] R. Brody, Compact manifolds and hyperbolicity, Trans. Amer. Math. Soc. 235 (1978), 213-219.

[2] K. Diederich and N. Sibony, Strange complex structures on Euclidean space, J. Reine Angew. Math. 311/312 (1979), 397-407.

[3] G. Fischer, Complex Analytic Geometry, Lecture Notes in Math. 538, Springer, 1976.

[4] A. Hirschowitz, Pseudoconvexité au-dessus d'espaces plus ou moins homogènes, Invent. Math. 26 (1974), 303-322. 
[5] S. Kobayashi, Hyperbolic Manifolds and Holomorphic Mappings, Dekker, 1970.

[6] - Intrinsic distances, measures and geometric function theory, Bull. Amer. Math. Soc. 82 (1976), 357-416.

[7] S. Lang, Introduction to Complex Hyperbolic Spaces, Springer, 1987.

[8] P. Mazet, Analytic Sets in Locally Convex Spaces, North-Holland Math. Stud. 121, North-Holland, 1987.

[9] J. P. Ramis, Sous-ensembles Analytiques d'une Variété Banachique Complexe, Ergeb. Math. Grenzgeb. 53, Springer, 1970.

[10] B. Shabat, Introduction to Complex Analysis, Part II, Functions of Several Variables, Transl. Math. Monographs 110, Amer. Math. Soc., 1992.

[11] B. Shiffman, Extension of holomorphic maps into hermitian manifolds, Math. Ann. 194 (1971), 249-258.

[12] N. Sibony, Prolongement des fonctions holomorphes bornées et métrique de Carathéodory, Invent. Math. 29 (1975), 205-230.

[13] B. D. Tac, Extending holomorphic maps in infinite dimensions, Ann. Polon. Math. 54 (1991), 241-253.

[14] D. D. Thai, Royden-Kobayashi pseudometric and tautness of normalizations of complex spaces, Boll. Un. Mat. Ital. A (7) 5 (1991), 147-156.

[15] H. Toruńczyk, Smooth partitions of unity on some non-separable Banach spaces, Studia Math. 66 (1973), 44-51.

[16] T. Urata, The hyperbolicity of complex analytic spaces, Bull. Aichi Univ. of Education 31 (1982), 65-75.

[17] E. Vesentini, Invariant distances and invariant differential metrics in locally convex spaces, in: Spectral Theory, Banach Center Publ. 8, PWN-Polish Sci. Publ., Warszawa, 1982, 493-512.

[18] M. Zaŭdenberg, The Picard theorem and hyperbolicity, Siberian Math. J. 24 (1983), 858-867 (English transl.).

[19] M. Zaŭdenberg, S. Krĕ̌n, P. Kuchment and A. Pankov, Banach bundles and linear operators, Uspekhi Mat. Nauk 30 (1975), no. 5, 101-157 (in Russian).

Department of Mathematics

Institute of Pedagogy of Hanoi 1

Cau giay, Tu liem

Ha noi, Vietnam 